\title{
40-Gb/s Optical Switching and Wavelength Multicasting in A Two-Pump Parametric Device
}

\author{
Q. Lin, R. Jiang, C. F. Marki, C. J. McKinstrie, R. Jopson, J. Ford, G. P. Agrawal, and S. Radic
}

\begin{abstract}
A two-pump parametric architecture is used to demonstrate all-optical packet and bit-level switching at $\mathrm{OC}-768$ data rates. We show that a switched signal can be simultaneously replicated in four parametric bands with arbitrary conjugate states. The conversion efficiencies and extinction ratios are controlled by simple pump-spectral positioning. The use of subrate pump switching is analyzed and demonstrated using arbitrary 40-Gb/s sequences.
\end{abstract}

Index Terms-Highly nonlinear fiber (HNLF), optical switching, parametric processing, wavelength casting.

H IGH-CONFINEMENT silica fibers provide virtually unlimited optical processing speed with low noise [1]. Degenerate (one-pump) four-photon mixing (FPM) in silica has been successfully used in a range of applications including wavelength conversion, pulse generation, optical sampling, and signal conjugation. A multiple-pump parametric configuration has recently been used in an effort to increase the bandwidth and efficiency of all-optical processing applications [2]-[5]. The two-pump parametric (TPP) architecture introduces additional degrees of design freedom because the wavelength, power, and state of polarization (SOP) of each pump can be independently controlled. Unlike one-pump devices, TPP devices have four operational bands. Since all four wavebands can be used in practice, the spectral range and operational functionality is enhanced considerably [2].

The multibanded nature of two-pump devices has been previously used in $10-\mathrm{Gb} / \mathrm{s}$ switching and multicasting [5]. While high-confinement fiber allows for terabits/second switching rates, bit-level manipulation is ultimately limited by the speed of pump control which needs to be comparable to input data rates. This letter demonstrates, for the first time to our knowledge, multiple-band $40-\mathrm{Gb} / \mathrm{s}$ switching with a conversion efficiency of at least $21.2 \mathrm{~dB}$ and an extinction ratio exceeding $25.0 \mathrm{~dB}$. The TPP configuration was used to achieve multicasting and $40-\mathrm{Gb} / \mathrm{s}$ bit-level signal processing within four operational bands. More importantly, the feasibility of subrate pump control was demonstrated for OC-768 signal processing.

Our two-pump experimental setup is shown in Fig. 1. Two continuous-wave $(\mathrm{CW})$ tunable lasers $\left(\lambda_{1}=1568.5 \mathrm{~nm}\right.$ and

\footnotetext{
Manuscript received June 7, 2005; revised August 10, 2005. This work was supported by the National Science Foundation under Grant ECS-0320816 and Grant ECS-0334982.

Q. Lin and G. P. Agrawal are with the Institute of Optics, University of Rochester, Rochester, NY 14627 USA.

R. Jiang, C. F. Marki, J. Ford, and S. Radic are with the Department of Electrical and Computer Engineering, University of California, San Diego, CA 92093 USA.

C. J. McKinstrie and R. Jopson are with the Bell Laboratories, Lucent Technologies, Holmdel, NJ 07733 USA.

Digital Object Identifier 10.1109/LPT.2005.858155
}

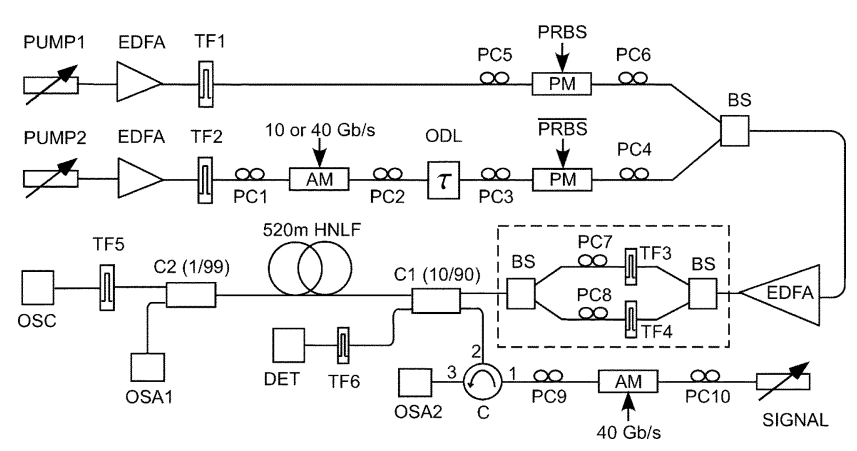

Fig. 1. Experimental setup. TF: tunable filter. PM: phase modulator. AM: amplitude modulator. PC: polarization controller. ODL: optical delay line. OSA: optical spectrum analyzer. OSC: oscilloscope. BS: band splitter. C1: 10/90 coupler. C2: 1/99 coupler. C: circulator. DET: detector; it can be either an OSA, an OSC, or a power meter used for characterizing the input signal, $C$ - or $L$-band pump. OSA2 is used for monitoring Brillouin scattering of the pumps.

$\left.\lambda_{2}=1598.0 \mathrm{~nm}\right)$ served as the pump seeds, and were amplified and filtered by two 0.25 -nm filters (TF1 and TF2) to reduce the amplified spontaneous emission (ASE) prior to the booster amplifier. The anomalous pump was controlled using a programmed nonreturn-to-zero (NRZ) bit sequence in both packet and bit-level switching experiments. A tunable optical delay line was used to adjust the relative delay between the pump and the signal. The two pumps were counterphased using a $5-\mathrm{Gb} / \mathrm{s}$ pseudorandom bit sequence to suppress stimulated Brillioun scattering generated inside both the booster amplifier [erbium-doped fiber amplifier (EDFA)] and the highly nonlinear fiber (HNLF). The EDFA booster provided an average output power of $2.3 \mathrm{~W}$, measured prior to the ASE-filtering section (dashed box in Fig. 1). The booster ASE was filtered out using two 1-nm tunable filters (TF3 and TF4) in order to guarantee pump spectral purity [1] greater than $75 \mathrm{~dB}$ (measured within 0.2-nm bandwidth). A third tunable $\mathrm{CW}$ laser served as the signal. It was modulated using a $40-\mathrm{Gb} / \mathrm{s}$ NRZ pattern and was inserted into the HNLF through a 10/90 coupler. PC7, PC8, and PC9 were all adjusted to achieve a copolarized SOP for the pumps and the signal inside HNLF. The 520-m HNLF used in the experiments had an effective area of $11 \mu \mathrm{m}^{2}$, a dispersion slope of $0.025 \mathrm{ps} /\left(\mathrm{nm}^{2} \cdot \mathrm{km}\right)$, and a zero-dispersion wavelength of $1582 \mathrm{~nm}$. The four sidebands were individually selected using a 2-nm-wide optical filter (TF5) and observed using a fast oscilloscope (37-GHz response).

As a first goal of this study, we investigated the performance of arbitrary-bit-sequence (packet) extraction from the input OC-768 stream. Unlike the previously reported result [5], the switching of the OC-768 sequence required precise control beyond that of the OC-192 rate. Indeed, while the length of the switched sequence ( $\sim 1000 \mathrm{ps}$, see experiments below) allows 


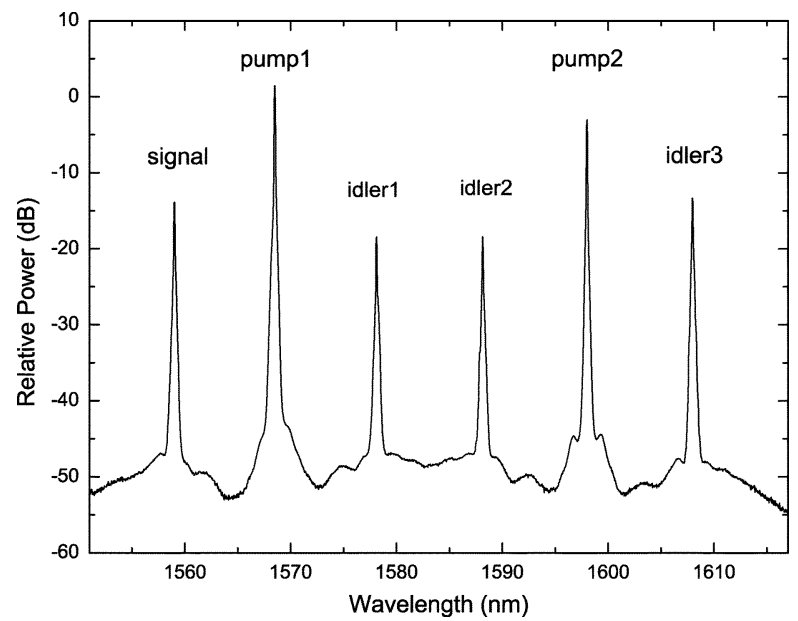

Fig. 2. Optical spectrum measured at the output of HNLF, recorded with $0.2-\mathrm{nm}$ resolution when a $1559-\mathrm{nm}$ signal is launched. Other details are given in the text.

for slow pump control, extraction with zero guardband does require speed comparable to the input data rate (OC-768) or even faster. However, parametric generation inside an HNLF helps to reduce significantly such requirement on the pumps because the output signal/idler powers are related to the pumps by an exponential gain function. For simplicity, if the parametric process is assumed to be dominated by a single phase-matched FPM process, the true switching rise-fall time is determined by the parametric response as $\exp \left[2 \gamma L P_{\text {eff }}(\tau)\right] / 4$, where $P_{\text {eff }}(\tau)$ represents the effective pump power, defined as $2 \sqrt{P_{1} P_{2}(\tau)}$ for the nondegenerate (two-pump) interaction and $P(\tau)$ for the degenerate (one-pump) interaction; $L$ is the HNLF length and $\gamma$ is the nonlinear parameter. For a parametric gain of 20-30 dB, this formula yields a parametric response two to four times faster than the corresponding pump rise-fall time. In general, the single-pump configuration exhibits faster response [6]. However, dual-pumping provides more functional sidebands and, thus, enables wavelength multicasting. The parametric rise-fall time associated with $10-\mathrm{Gb} / \mathrm{s}$ pump control ( $\sim 25$ ps rise-fall time) was indistinguishable from that of the $40-\mathrm{Gb} / \mathrm{s}$ sequence in practice. This indicates that $10-\mathrm{Gb} / \mathrm{s} \mathrm{pump}$ control could be effectively used to achieve the practical packet switching at OC-768 rates. Consequently, it allowed a control of the anomalous pump by a $10-\mathrm{Gb} / \mathrm{s}$, rather than a $40-\mathrm{Gb} / \mathrm{s}$, programmed sequence.

To demonstrate OC-768 packet switching, the long-wavelength pump was modulated using a 1-ns-long rectangular pulse (composed of ten successive logical ones at $10 \mathrm{~Gb} / \mathrm{s}$, corresponding to 40 bits at $40 \mathrm{~Gb} / \mathrm{s}$ ) in order to extract a selected sequence from the data stream of the small input signal $\left(P_{\text {in }}=-14 \mathrm{dBm}\right)$. The average pump powers launched into the HNLF were 545 and $155 \mathrm{~mW}$ for the normal ( $C$-band) and anomalous ( $L$-band) pumps, respectively. The TPP spectrum is shown in Fig. 2, illustrating three new switched wavelengths at $1578.1,1588.1$, and $1608.0 \mathrm{~nm}$. The input signal at $1559.0 \mathrm{~nm}$ is simultaneously amplified by $25.2 \mathrm{~dB}$ ("ON-OFF" value, measured at the HNLF output) and multicasted with conversion efficiencies of 21.2, 21.4, and $25.9 \mathrm{~dB}$, respectively, covering a spectral range of $49 \mathrm{~nm}$. The corresponding $0.2-\mathrm{nm}$ optical signal-to-noise ratios were measured to be $33.6 \mathrm{~dB}$ $(1559.0 \mathrm{~nm}), 28.5 \mathrm{~dB}(1578.1 \mathrm{~nm}), 28.9 \mathrm{~dB}(1588.1 \mathrm{~nm})$, and

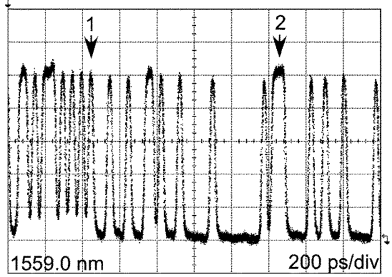

(a)

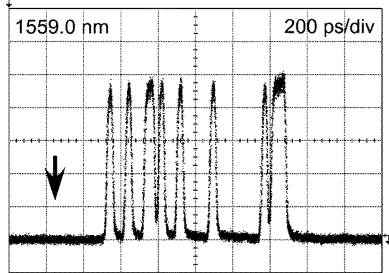

(c)

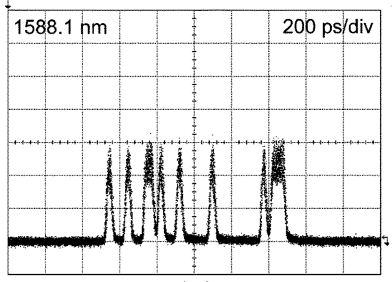

(e)

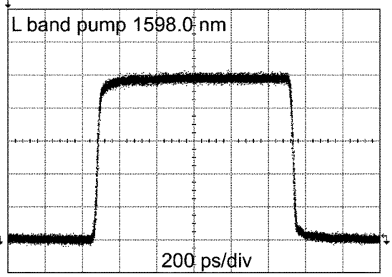

(b)

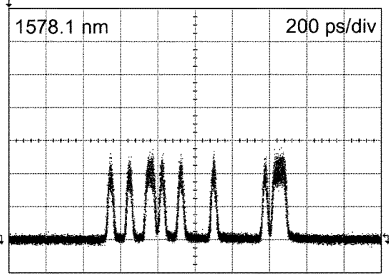

(d)

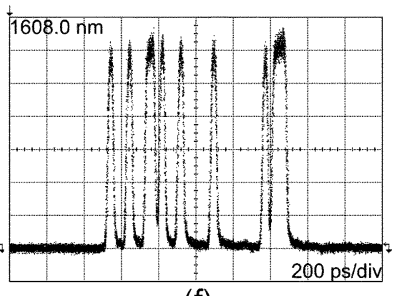

(f)
Fig. 3. Temporal waveforms showing packet switching. A fixed input level was used in the oscilloscope for all signal/idlers waves. (a) Input $1559.0-\mathrm{nm}$ signal; (b) $L$-band pump; (c) amplified signal; (d) switched 1578.1-nm idler; (e) switched 1588.1-nm idler; (f) switched 1608.0-nm idler.

$34.8 \mathrm{~dB}(1608.0 \mathrm{~nm})$. The absence of higher order wave [4] in the vicinity of the switched sidebands confirms the linear TPP operation required for crosstalk-free waveband switching.

The temporal waveforms of the four sidebands and the $L$-band pump are shown in Fig. 3. The input signal had a mark density of $1 / 2$ [Fig. 3(a)], with the extinction ratio limited by the electronic driver used for the $40-\mathrm{GHz}$ AM modulator. Fig. 3(b) shows the square waveform imposed on anomalous pump used for OC-768 sequence extraction. Switched packets were obtained from all four parametric bands. That at the signal wavelength $(1559.0 \mathrm{~nm})$ is shown in Fig. 3(c), and the corresponding idler packets at 1578.1, 1588.1, and $1608.0 \mathrm{~nm}$ are shown in Fig. 3(d)-(f), respectively. Switched packet traces were recorded by a simple tuning of the output (TF5) filter, since high power $(>5 \mathrm{dBm})$ in any of the four streams eliminated the need for postamplification. The switching is realized with low noise and high extinction ratio: No artifacts were observed in the immediate vicinity outside the switched window [indicated by an arrow in Fig. 3(c)], in spite of the dense bit content carried by the input signal in this region. The higher noise levels seen with innerband packets (1578.1 and $1588.1 \mathrm{~nm}$ ) are attributed to lower conversion efficiencies and the fixed input level used with the oscilloscope; no attempt was made to adjust the power levels to reduce the receiver noise.

Physically, a high extinction ratio stems from the exponential dependence of the parametric gain on the pump powers. For example, in the phase-matched region, the parametric gain (in decibels) produced via nondegenerate FPM scales with pump powers as $\sqrt{P_{1} P_{2}}$. In contrast, neither is the signal amplified nor are the idlers created in the absence of $L$-band pump, since the $C$-band $\mathrm{CW}$ pump located in the normal-dispersion region 


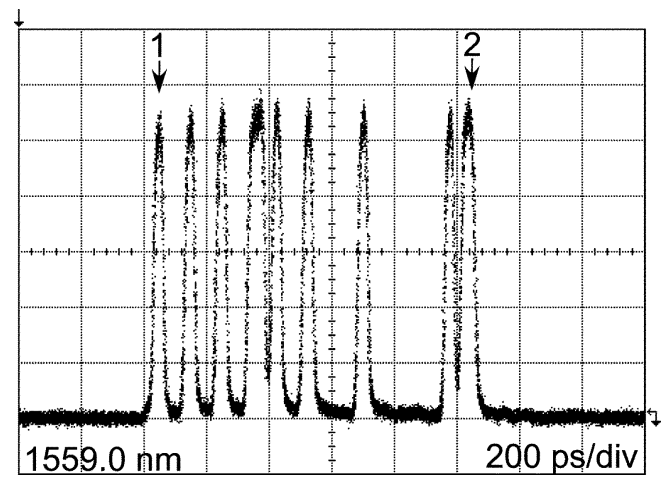

Fig. 4. Amplified 1-ns packet of the 1559.0-nm signal when the $L$-band pump is temporally shifted in such a way that its leading edge coincides with the signal bit marked by arrow 1 in Fig. 4(a).

produces negligible FPM. Clearly, high parametric gain guarantees switching with high extinction ratios in all four sidebands.

The TPP process allows for arbitrarily long packets to be switched simply by varying the time interval in which both pumps are simultaneously present. More importantly, the effective compression of rise-fall time allows the use of slow pump control to achieve precise bit control. Indeed, the pump rise-fall time of $25 \mathrm{ps}$ in this experiment was sufficient to achieve zeroguardband packet switching. To demonstrate this capability, the anomalous pump switching window was shifted to cover the selected "1" bit, as indicated by Arrow 1 in Fig. 3(a). The switched packet is shown in Fig. 4: It can be seen clearly that the selected "1" was switched in, with the preceding bits eliminated completely. At the same time, the last bit in the sequence, "1" indicated by Arrow 2, was steeply carved out from the successive logical ones. Clearly, the instantaneous parametric response of FWM dramatically reduces the requirement of the guard time for packet switching [7] and, thus, would help to increase the efficiency of optical processing schemes required in transparent networks.

As a second goal of this study, we demonstrated optical switching at the bit level. The input signal is modulated with a $40-\mathrm{Gb} / \mathrm{s}$ NRZ bit pattern, as shown in Fig. 5(a) (other bit patterns can also be used). The anomalous pump was controlled by an isolated bit, as illustrated in Fig. 5(b). The pump was used to select the bits of a logical " 0 " and a logical "1" from the input sequence, as indicated by arrows in Fig. 5(a). High extinction ratio is demonstrated by comparing the contrast between switched " 0 " [inset in Fig. 5(c)] and switched " 1 " [Fig. 5(c)]. When the selected bit represents a high logical level, the signal is simultaneously amplified and replicated to three idler waves. Bit-level wavelength casting at $40 \mathrm{~Gb} / \mathrm{s}$ is illustrated in Fig. 5(d), corresponding to translation of the selected bit " 1 " in the sequence to the outer parametric band (1559 $\rightarrow$ $1608 \mathrm{~nm}$ ). Similar switched bits (with smaller amplitudes) were obtained for the two inner bands.

The switched bits had full-widths at half-maximum of $20 \mathrm{ps}$ $(1559.0 \mathrm{~nm}), 17 \mathrm{ps}(1578.1 \mathrm{~nm}), 16 \mathrm{ps}(1588.1 \mathrm{~nm})$, and $20 \mathrm{ps}$ $(1608.0 \mathrm{~nm})$, clearly indicating compression with respect to the original bits in the input sequence. The two inner bands had higher compression ratios, as expected by the different contributions of degenerate and nondegenerate FPM. Such pulse compression can be used to reshape the switched pulse and provide

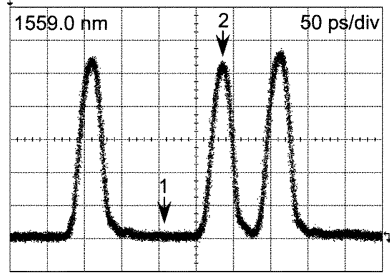

(a)

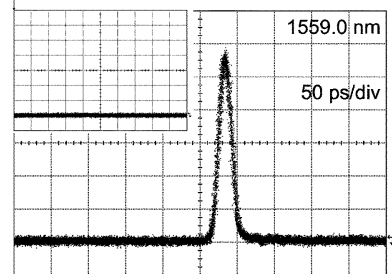

(c)

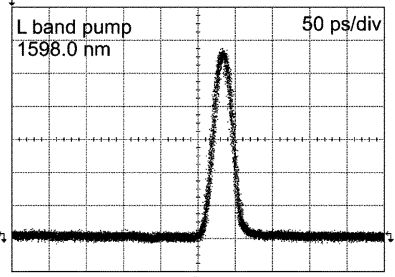

(b)

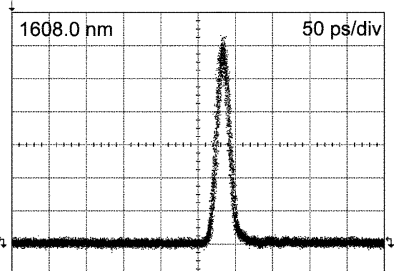

(d)
Fig. 5. Temporal waveforms showing bit-level switching. (a) Input 1559.0-nm signal; (b) $L$-band pump; (c) amplified signal bit when the pump bit is located at Position 2. The inset shows the signal waveform when the pump bit is located at Position 1. (d) Switched bit for the outer-band idler at $1608.0 \mathrm{~nm}$.

an efficient way for signal regeneration within the routing node itself.

In conclusion, we have demonstrated optical switching with wavelength conversion at $40 \mathrm{~Gb} / \mathrm{s}$ by using a TPP architecture. The scheme realizes packet switching for arbitrary packet lengths with negligible guard-time requirements and subrate pump controls. The experiment validated TPP performance that was sufficient to switch individual bits at $40 \mathrm{~Gb} / \mathrm{s}$, while maintaining high extinction ratios and conversion efficiencies above $20 \mathrm{~dB}$. We note that the architecture also possesses an inherent ability to selectively conjugate a switched packets or bit sequences, thereby enabling the possibility for applications involving simultaneous high-speed switching and transmission-impairment mitigation.

\section{REFERENCES}

[1] J. Hansryd, P. A. Andrekson, M. Westlund, J. Li, and P. O. Hedekvist, "Fiber-based optical parametric amplifiers and their applications," IEEE J. Sel. Topics Quantum Electron., vol. 8, no. 3, pp. 506-520, May/Jun. 2002.

[2] T. Tanemura, C. S. Goh, K. Kikuchi, and S. Y. Set, "Highly efficient arbitrary wavelength conversion within entire $C$-band based on nondegenerate fiber four-wave mixing," IEEE Photon. Technol. Lett., vol. 16, no. 2, pp. 551-553, Feb. 2004.

[3] K. Uesaka, K. K. Y. Wong, M. E. Marhic, and L. G. Kazovsky, "Wavelength exchange in a highly nonlinear dispersion-shifted fiber: theory and experiments," IEEE J. Sel. Topics Quantum Electron., vol. 8, no. 3, pp. 560-568, May/Jun. 2002.

[4] S. Radic, C. J. McKinstrie, R. M. Jopson, J. C. Centanni, and A. R. Chraplyvy, "All-optical regeneration in one- and two-pump parametric amplifiers using highly nonlinear optical fiber," IEEE Photon. Technol. Lett., vol. 15, no. 7, pp. 957-959, Jul. 2003.

[5] S. Radic, C. J. McKinstrie, R. M. Jopson, A. H. Gnauck, J. C. Centanni, and A. R. Chraplyvy, "Multiple-band bit-level switching in two-pump fiber parametric devices," IEEE Photon. Technol. Lett., vol. 16, no. 3, pp. 852-854, Mar. 2004.

[6] T. Torounidis, M. Westlund, H. Sunnerud, B. E. Olsson, and P. A. Andrekson, "Signal generation and transmission at 40, 80, and $160 \mathrm{~Gb} / \mathrm{s}$ using a fiber-optical parametric pulse source," IEEE Photon. Technol. Lett., vol. 17, no. 2, pp. 312-314, Feb. 2005.

[7] K. Onohara, Y. Awaji, N. Wada, F. Kubota, and K. Kitayama, "Agile and highly efficient wavelength conversion using highly nonlinear fiber for optical code-labeled packets," IEEE Photon. Technol. Lett., vol. 17, no. 3, pp. 627-629, Mar. 2005. 